\title{
Osteonecrosis of carpal bones in systemic sclerosis
}

\author{
Alexandra Chitac ${ }^{1}$, Codrina Ancuta ${ }^{2,3}$ \\ ${ }^{1}$ Rheumatology Department, "Regina Maria" Medical Campus, lasi, Romania \\ 2"Grigore T. Popa" University of Medicine and Pharmacy, lasi, Romania \\ ${ }^{3}$ 2nd Rheumatology Department, Clinical Rehabilitation Hospital, lasi, Romania
}

\begin{abstract}
Background. Systemic sclerosis (SSc) is a rare chronic disease, with unknown aetiology and complex pathogenesis. The hand is often implied in the main clinical findings, being affected primarily by the vascular component (Raynaud phenomenon, digital ulcers). One of the possible complications of the hand vasculopathy is osteonecrosis (ON) of the carpal bones. In these cases, the lunate is the most frequently affected, followed by the scaphoid. In the ON process, not only the vascular supply is important, but the nearby mechanical factors and anatomic variants of the wrist. Our objective was to reveal the most important aspects regarding the ON of the carpal bones in patients with SSc.
\end{abstract}

Methods. A systematic literature review was performed through July 31, 2021 on Pubmed and Cochrane databases. The eligible articles were read in full text and were included in this paper, in the absence of exclusion criteria and after consensus between two reviewers.

Results. Ten articles met the inclusion criteria, their main results being described in this review. In all studies, ON of the lunate and the scaphoid is associated with advanced SSC and severe Raynaud phenomenon.

Conclusions. In conclusion, carpal ON is a rare complication of SSc, especially of the diffuse type. There is limited data on this condition, its prevalence being difficult to estimate due to the lack of symptoms.

Keywords: systemic sclerosis, carpal bones, osteonecrosis, disability

\section{INTRODUCTION}

Classified as an orphan disease, systemic sclerosis (SSc) is a rare chronic autoimmune condition, with a still unknown aetiology and a complex pathogenesis $(1,2)$. Three main pathobiological pathways are essential for the disease including autoimmune vascular damage, skin and internal organs fibrosis and specific immune abnormalities, resulting in a wide spectrum of clinical manifestations ranging from peripheral digital vasculopathy expressed as Raynaud phenomenon and digital ulcers, to multiple-organ involvement (lung, renal, cardiac, gastrointestinal) (3).

Osteonecrosis (ON), avascular or aseptic bone necrosis, remains a common complication and a major cause of disability among rheumatic conditions, process in which there is a decreased blood supply to the bone, resulting in the necrosis of the bony tissue. ON generally involves the weight bear- ing joints, particularly the femoral head, in young, physically active men (4), but there are cases in which the non-weight bearing joins are affected, most commonly the carpal bones (5). Usually, $\mathrm{ON}$ is localized on a single anatomical site, but less frequent, multifocal ON is described, most of the cases being related to glucocorticoids therapy and/or autoimmune diseases (6). The underlying mechanisms are complex and well-studied especially in systemic lupus erythematosus (SLE) and antiphospholipid syndrome (APS) (7), comprising (i) a direct injury of the bone vessels as described in fracture and trauma; (ii) fatty transformation of the bone marrow, classically shown in glucocorticoid-treated patients and alcohol abuse; (iii) vascular endothelial-cell injury as reported in rheumatic autoimmune conditions such as lupus, scleroderma and vasculitis; and (iv) occlusion of the vessels typically found in hypercoagulable states associated with neoplasia and sickle cell disease (8). 
Although different authors have reported osteonecrosis in patients diagnosed with systemic sclerosis, there is limited data on this topic as its prevalence is difficult to estimate due to the lack of symptoms (8). The aim of this paper is to reveal the most important aspects regarding the $\mathrm{ON}$ of the carpal bones in SSc.

\section{METHODS}

We performed a comprehensive search in $\mathrm{Pu}$ bMed and Cochrane Library databases through July 31th, 2021. The keywords used were "osteonecrosis", "systemic sclerosis" and "carpal bones". We applied no restriction for language, year or type of publication.

Duplicate studies were excluded and the remaining articles were independently reviewed by two reviewers, as follows: first, titles and abstracts were analysed according to the inclusion criteria; all the scientific papers containing data on the association between SSc and ON of the carpal bones or the pathophysiology of ON in SSc patients were read in full text and selected to be included in this review. Study selection was guided by the aspects regarding the frequency and pathophysiology of the carpal ON in SSc, therefore all articles assessing these items were eligible for inclusion. Articles discussing the relationship between SSc and ON in other anatomical sites were excluded, as well as those in the preprint form. Studies included in this review were case-reports systematic reviews and one retrospective study, all of them referring to the lunate or scaphoid ON. No study on ON of the other carpal bones was found.

\section{RESULTS}

Overall, 97 publications were read: 89 in PubMed and 8 in Cochrane Library. Of these, 63 were excluded after abstract analysis, 3 were duplicate studies and 2 were not available online. From the remaining, 24 articles were excluded after full text review for not meeting the inclusion criteria and another 5 additional papers were included after reviewing the references. At the end of the review process, 10 articles were included in this scientific paper (Figure 1).

Characteristics of the selected publications and the main results are listed in Table 1 . All articles revealed a strong relationship between advanced SSc, with severe vascular manifestations (Raynaud phenomenon, digital ulcers) and lunate or scaphoid ON.

\section{DISCUSSION}

Carpal bone osteonecrosis and particularly lunate involvement remains a potential complication of systemic sclerosis emphasized by different papers (9). Although several mechanisms have been proposed, it seems that vascular damage and subsequent endothelial injury is the essential pathobiological pathway involved in ON secondary to SSc. Furthermore, a strong association between the severity of Raynaud phenomenon, presence of active digital ulcers and the lunate involvement is typically described, particularly in advanced SSc $(10,11)$.

We will further focus on osteonecrosis and its main subtypes in patients with SSc, after a brief remember of the carpal bone anatomy.

\section{Anatomy of the carpal bones}

The skeleton of the wrist is designed as two rows of small bones, each one consisting of four; from the radial to the ulnar side, the proximal row includes the scaphoid, the lunate, the triquetrum and the pisiform bone, while the distal row comprises the trapezium, the trapezoid, the capitate and the hamate

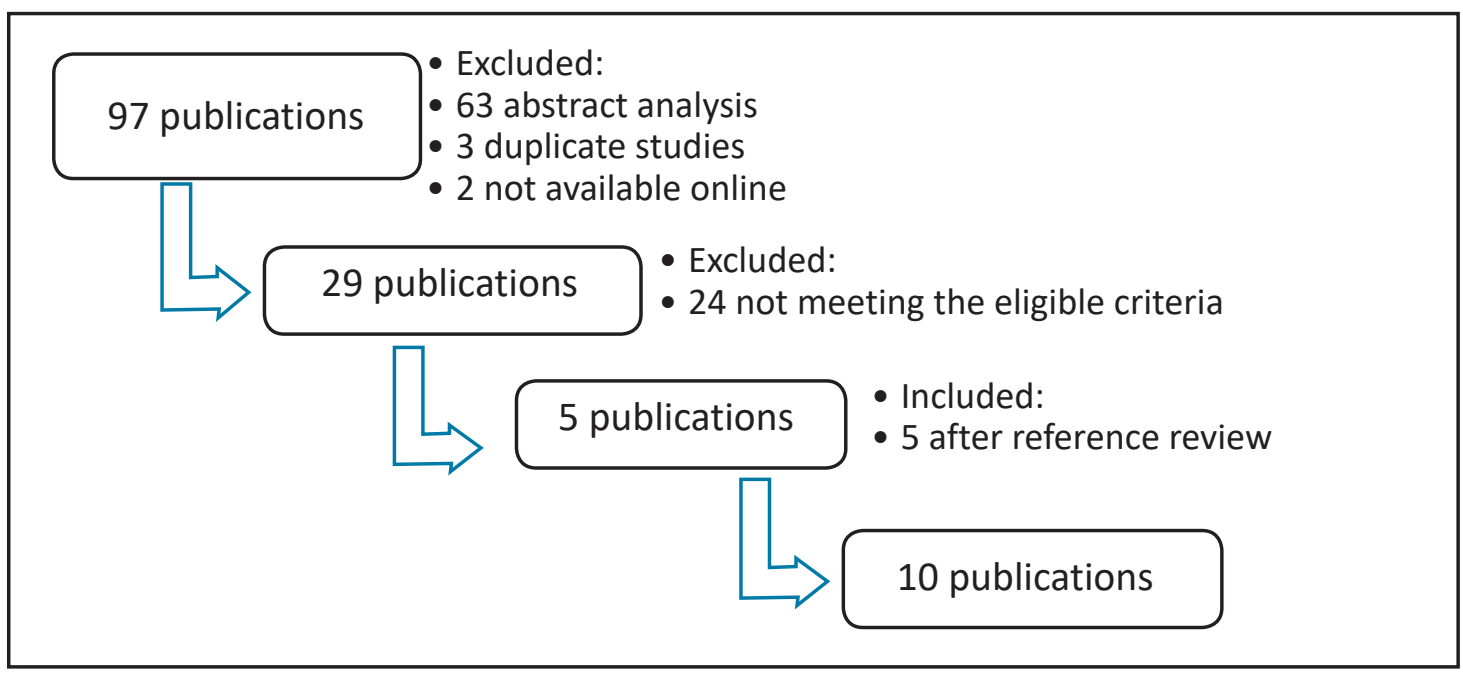

FIGURE 1. Flowchart with article selection 
TABLE 1. Characteristics and results of the selected articles

\begin{tabular}{|c|c|c|c|}
\hline Author & Year & Type of article & Main conclusion \\
\hline Jeong et al. (5) & 2018 & $\begin{array}{l}\text { Retrospective } \\
\text { study }\end{array}$ & $\begin{array}{l}\text { There are cases of SSc in which the non-weight bearing joins are affected, most } \\
\text { commonly the carpal bones. }\end{array}$ \\
\hline Basyal et al. (6) & 2021 & Case-report & $\begin{array}{l}\text { In SSc, as well as other autoimmune diseases, the endothelial injury is one of } \\
\text { the main underlying mechanisms of ON. }\end{array}$ \\
\hline Rennie el al. (11) & 1999 & Case-report & $\begin{array}{l}\text { Advanced SSc, with severe Raynaud phenomenon and, in some cases, negative } \\
\text { ulnar variant is associated with bilateral ON, diagnosed late in the evolution of } \\
\text { the disease. }\end{array}$ \\
\hline Bergman et al. (13) & 2021 & $\begin{array}{l}\text { Systematic } \\
\text { review }\end{array}$ & $\begin{array}{l}\text { The lunate is the most frequently involved carpal bone in the ON process in SSC } \\
\text { patients. }\end{array}$ \\
\hline Sandler et al. (14) & 2020 & Case-report & $\begin{array}{l}\text { Although the hand is often affected by SSc, lunate ON is an underappreciated } \\
\text { vascular complication, because of the lack of symptoms or the nonspecific wrist } \\
\text { pain. }\end{array}$ \\
\hline Frerix et al. (10) & 2016 & Case-report & $\begin{array}{l}\text { The involvement of the ulnar artery in the pathogenic process of avascular } \\
\text { necrosis revealed a strong relationship between the severity of the Raynaud } \\
\text { phenomenon, presence of digital ulcers and the lunate involvement. }\end{array}$ \\
\hline Hughes et al. (16) & 2020 & $\begin{array}{l}\text { Systematic } \\
\text { review }\end{array}$ & $\begin{array}{l}\text { There is a strong relationship between the vascular phenomenon in SSc and } \\
\text { lunate ON. }\end{array}$ \\
\hline $\begin{array}{l}\text { Zegarra-Mondragón } \\
\text { et al. (18) }\end{array}$ & 2019 & Case-report & $\begin{array}{l}\text { Despite treatment with low doses of corticosteroids, SSc patients do not } \\
\text { develop ON in common locations like the knee or the hip, carpal bones being } \\
\text { the only site affected. }\end{array}$ \\
\hline Kim et al. (20) & 2020 & Case-report & $\begin{array}{l}\text { A rare complication of the lunate } \mathrm{ON} \text { in SSc patients is extensor tendons } \\
\text { rupture the pathogenic mechanism being represented by abnormal alinement } \\
\text { of the carpal bones (in case of collapse), fragmentation of the lunate, associa- } \\
\text { ted amyloidosis and tendon fibrosis. }\end{array}$ \\
\hline Garg et al. (26) & 2011 & Case-report & The diagnostic of carpal ON is made almost exclusively by imaging technics. \\
\hline
\end{tabular}

(in the same order); for the practical purpose, we can consider each bone being a cuboid. The palmar and dorsal surfaces are non-articular, serving for the attachment of the ligaments that hold them together; also, these surfaces are the place where the nutrient foramen is (12). The carpal bones receive blood supply by two different patterns: the radial artery and the interosseous arteries (for the scaphoid, trapezium, lunate and capitate) and branches from the ulnar artery (lunate, triquetrum, hamate) (13).

\section{Osteonecrosis of the lunate (Kienböcks's disease or lunatomalacia)}

First mentioned by Peste in 1843 (14), lunate ON was fully described in 1910 by the Austrian radiologist Robert Kienböck who also named the disease after him and detailed it as a progressive debilitating condition, caused by the impaired blood supply of the lunate (15). The relationship with the SSc is recognized in many scientific papers, the lunate being the most frequently involved carpal bone in the ON process (9). Although the hand is often affected by SSc, lunate ON remains an underappreciated vascular complication, because of the lack of symptoms or the nonspecific wrist pain (16). Data regarding the involvement of the ulnar artery in the pathogenic process revealed a strong relationship between the severity of the Raynaud phenomenon, presence of digital ulcers and the lunate $\mathrm{ON}(10,17)$. Also, mechanical factors such as the length of the ulna, can be of great importance in the vascular component of the Kienböck's disease (KD). Hulten et al. described three anatomical variants of the relative length of radius and ulna (ulnar variances): neutral (both the ulnar and the radial joint surfaces are at the same level); positive (the ulna is longer than the radius and its joint surface projects more distally); negative (the ulna is shorter than the radius and its joint surface projects more proximally) (11). Usually, lunate ON is unilateral; advanced SSc, with severe Raynaud phenomenon and, in some cases, negative ulnar variant is associated with bilateral $\mathrm{ON}$, diagnosed late in the evolution of the disease (14). An article published in 2019 by Zegarra-Mondragón S et al. showed that the occlusion of the ulnar artery is common in patients with SSc, although it can be detected by Doppler ultrasound only in $21.5 \%$ of the cases. Additionally, the develop- 

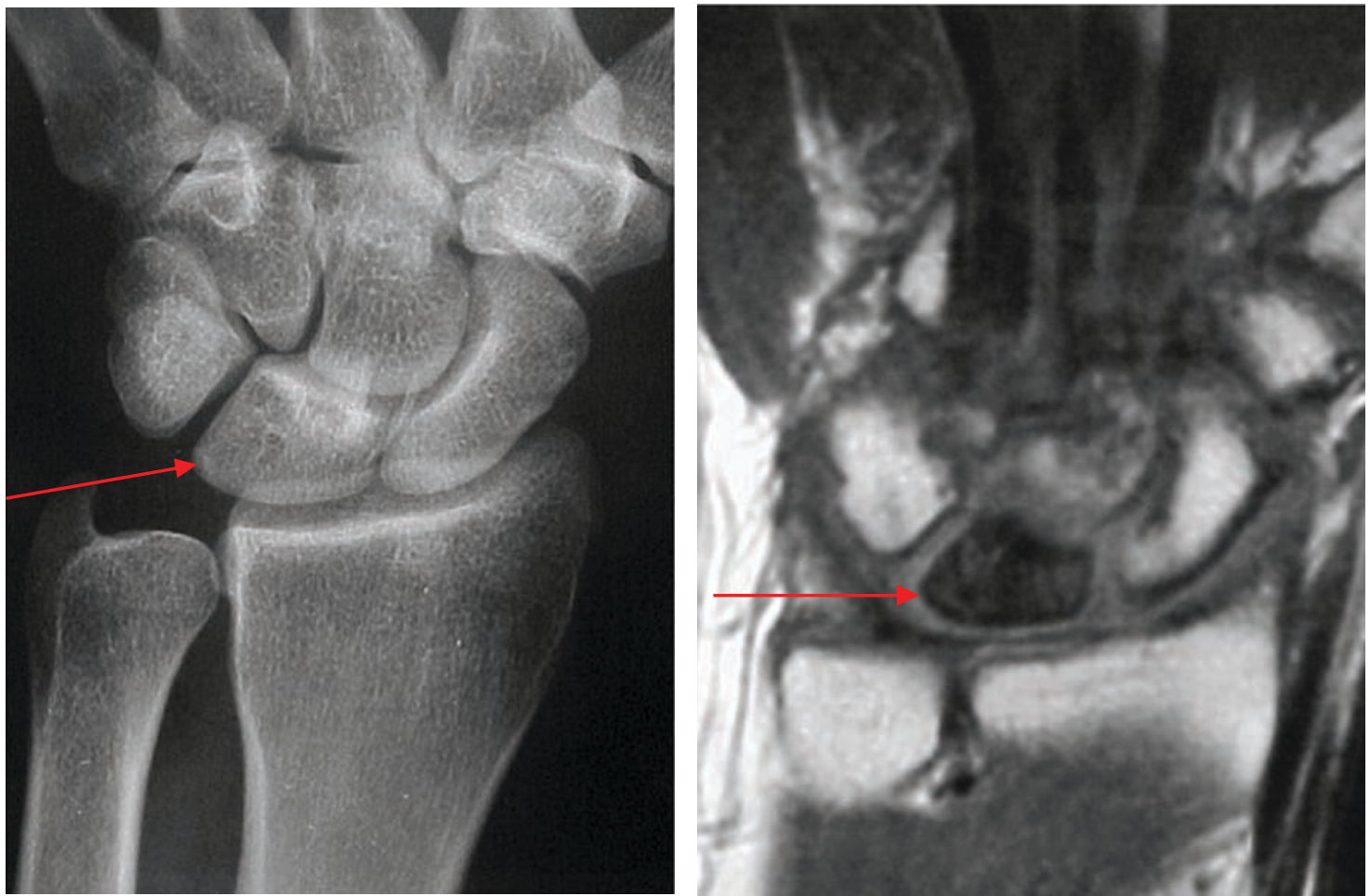

FIGURE 2. KD stage 1. Note normal aspect of the lunate on plain X-ray with signal loss on MRI - adapted after (15)

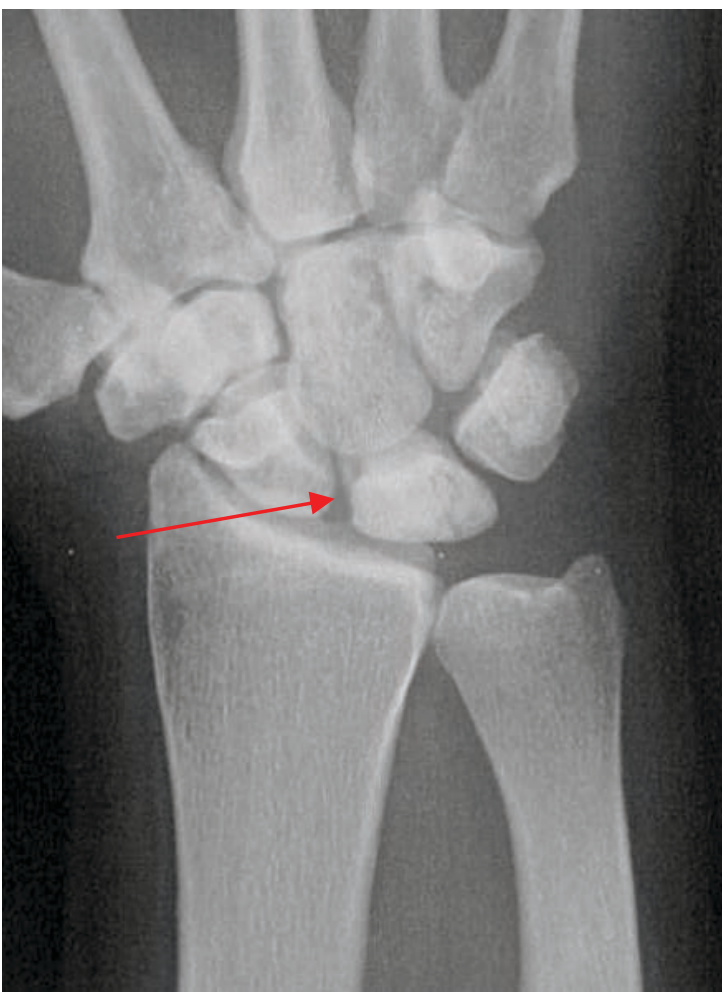

FIGURE 3. KD stage 2. Note the sclerotic aspect of the lunate, with normal joint surfaces - adapted after (15)

ment of $\mathrm{KD}$ in these patients is rarely associated with standard risk factors for lunate ON (anatomical variants, genetics, trauma). Moreover, despite treatment with low doses of corticosteroids, SSc patients do not develop ON in common locations such as the knee or the hip, carpal bones being the only affected anatomical site (18).
The diagnostic of KD is made by conventional radiography and magnetic resonance imaging (MRI). $\mathrm{X}$-rays are used for (i) the evaluation of risk factors for ON (ulnar variant, size of the lunate); (ii) bone modifications (diffuse sclerosis, the presence of cysts, fragmentation, collapse or signs of arthritis); and (iii) fractures, without being able to appreciate if it is an actual fracture line or the result of lunate collapse (15). There are many radiographic classifications of the $\mathrm{KD}$, but the one who has the most reproducibility and reliability is the Lichtman classification which recognized four stages: stage 1 -normal $\mathrm{X}$-ray, with signal changes on MRI (Figure 2); stage 2 - diffuse sclerotic aspect of the lunate on X-ray, with or without a fracture line, but with preserved joint surface (Figure 3); stage 3 - collapse of the lunate joint surface (3A - preserved carpal height; 3B - loss of carpal height, collapse of the wrist) (Figure 4,5); stage 4 - lunate collapse and arthritis of the radiocarpal and intercarpal joints (19).

MRI is helpful especially in stage 1, when changes are absent on X-rays. The most specific finding is signal loss in the entire area of the lunate and not localized on a single part (10).

A rare complication that can accompany lunate ON in SSc patients is extensor tendons rupture, especially in stage 3B or higher. The pathogenic mechanism is represented by abnormal alinement of the carpal bones (in case of collapse), fragmentation of the lunate, associated amyloidosis and tendon fibrosis (20).

The treatment of KD is exclusively surgical, depending on the disease stage. Thereby, in the first 


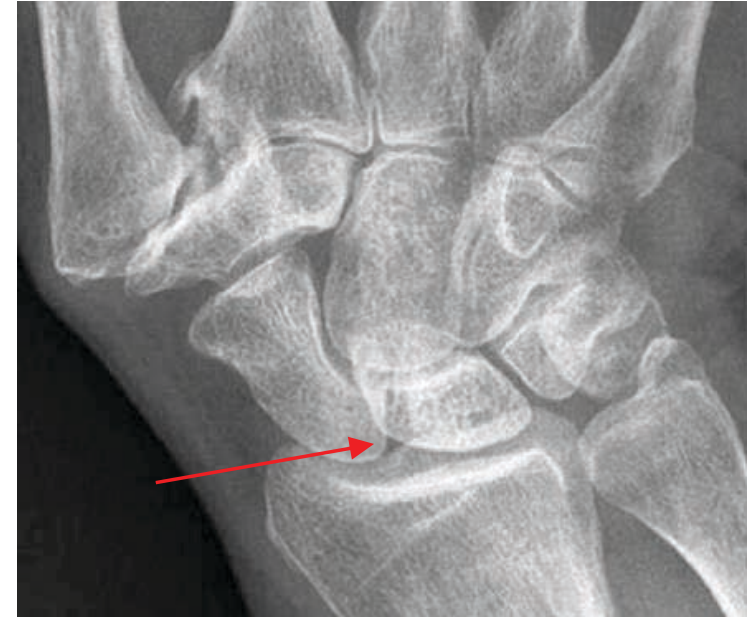

FIGURE 4. KD stage 3A. Note the lunate collapse with normal carpal height - adapted after (10)

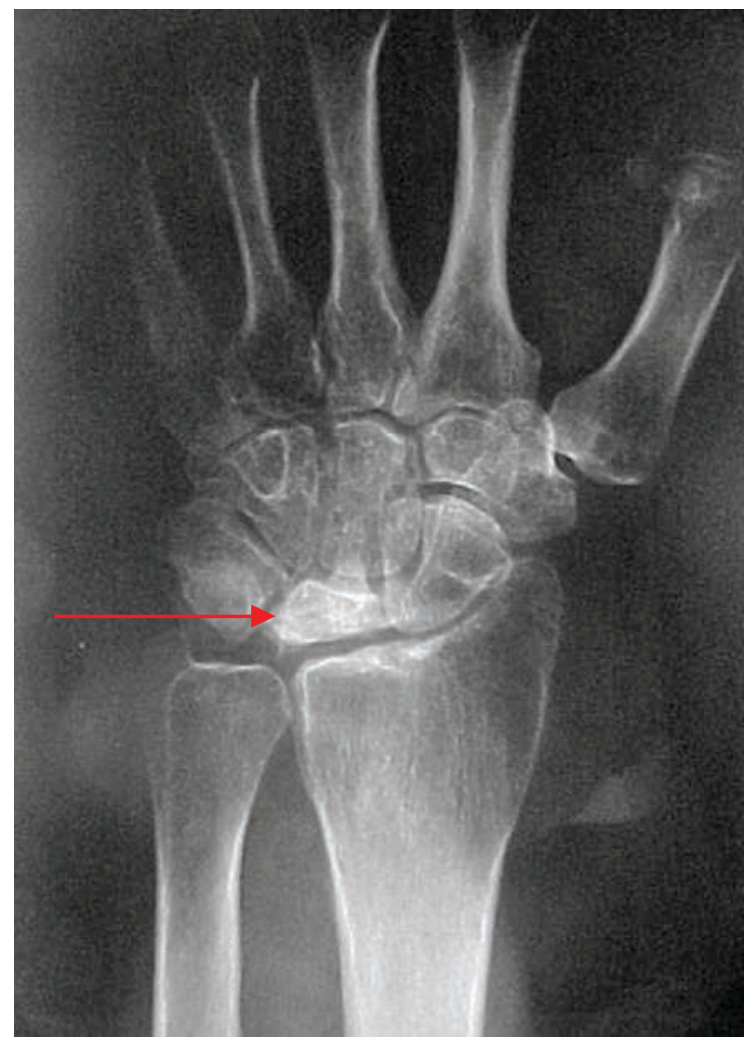

FIGURE 5. KD stage 3B. Note the collapse of the wrist adapted after (15)

stage, immobilisation is preferred. In stage 2 and $3 \mathrm{~A}$, arthroscopy or radial osteotomy are performed. Bone graft is the therapeutical option in stage 3B, while in advanced cases radiocarpal arthroplasty is preferred $(21,22)$.

\section{Osteonecrosis of the scaphoid (Preiser's disease)}

Idiopathic ON of the scaphoid was also described in 1910 by Georg Preiser, as being a rare condition, associated with pain, swelling of the snuffbox and decreased range of motion in the wrist (23). The supposed underlying pathogenic mechanism is a decreased blood supply of the proximal pole of the bone, as it is provided by intraosseous vessels, and not true branches from the radial artery $(24,25)$. The scaphoid is the second most frequently involved carpal bone in ON in SSc patients (9).

The clinical findings are scarce, represented by nonspecific wrist pain, swelling of the wrist or only of the snuffbox, paraesthesia (when associated with carpal tunnel syndrome) (25), sometimes radial deviation of the carp and reduce range of motion, in late stages, when collapse occurs (23). These signs and symptoms are rarely assigned to ON in SSc patients; in these cases, other causes should be excluded, including radio-carpal synovitis and tissue fibrosis.

The diagnostic is almost exclusively made by imaging technics (26). Conventional X-rays are used to correctly identify the affected part of the bone (usually the proximal pole, sometimes the whole bone and rarely the distal pole) and the severity of ON. The latter is staged according to the Herbert-Lanzetta classification: stage 1 - normal aspect; stage 2 increased density of the proximal pole in contrast with the generalised osteoporotic aspect; stage 3 loss of the normal shape of the proximal pole, with/ without a fracture; stage 4 - scaphoid/carpal collapse.

MRI is used especially for the ON of the distal pole and shows low signal intensity in both T1 and T2-weighted images (27).

There is limited scientific data regarding other diagnostic methods besides imaging. Some studies recommend the intraoperative biopsy of the scaphoid, a difficult and invasive procedure though (28).

The treatment is different for the pre-collapse faze (stages 2, 3) and the advanced ON (collapse, stage 4). In the first case, the therapeutical options include immobilization, arthroscopic debridement, bone grafting or silicone replacement of the affected part of the scaphoid. In stage 4, treatment is represented by bone excision or total wrist fusion (29).

\section{CONCLUSIONS}

ON of the carpal bones is a rare complication of the SSc, especially of the diffuse type. It is related to rapidly progressive forms, with severe Raynaud phenomenon and digital ulcers. Usually, the diagnostic is late, due to the lack of symptoms or the nonspecific pain; on the other hand, when clinical signs are present, they are attributed to other modifications, such as arthritis. The early diagnostic of carpal ON is of great importance and can spare the patient from disability and invasive surgical treatment. 


\section{REFERENCES}

1. Adigun R, Goyal A, Bansal P, Hariz A. Systemic Sclerosis. In: Treasure Island (FL): StatPearls Publishing; 2021.

2. Bergamasco A, Hartmann N, Wallace L, Verpillat P. Epidemiology of systemic sclerosis and systemic sclerosis-associated interstitial lung disease. Clinical Epidemiology. 2019;11:257273.

3. Strange G, Nash P. The manifestations of vasculopathy in systemic sclerosis and its evidence-based therapy. Int J Rheum Dis. 2009;12(3):192-206.

4. Mankin HJ. Nontraumatic necrosis of bone (osteonecrosis). N Engl J Med. 1992;326(22):1473-1479.

5. Jeong HJ, Kim D, Cho SK, et al. Clinical characteristics of multifocal osteonecrosis in Korean patients with rheumatic disease. Int J Rheum Dis. 2018;21(6):1301-1308.

6. Sun W, Shi Z, Gao F, et al. The pathogenesis of multifocal osteonecrosis. Sci Rep. 2016;6:29576.

7. Kamen DL, Alele JD. Skeletal manifestations of systemic autoimmune diseases. Curr Opin Endocrinol Diabetes Obes. 2010;17:540-3.

8. Basyal B, Bhandari B, Derk CT. Avascular necrosis in systemic sclerosis patients:a case-based review of demographics, presentation, and management. Clin Rheumatol. 2021;40(1): 399-405.

9. Bergman S, Petit A, Rabarin F, el al. Preiser's disease or avascular osteonecrosis of the scaphoid: An updated literature review. Hand Surg Rehabil. 2021;S2468-1229.

10. Frerix M, Kröger K, Szalay G, et al. Is osteonecrosis of the lunate bone an underestimated feature of systemic sclerosis? A case series of nine patients and review of literature. Semin Arthritis Rheum. 2016;45(4):446-454.

11. Hughes $M$, Allanore $Y$, Chung $L$, et al. Raynaud phenomenon and digital ulcers in systemic sclerosis. Nat Rev Rheumatol. 2020;16(4):208-221.

12. Tang A, Varacallo M. Anatomy, Shoulder and Upper Limb, Hand Carpal Bones. In:Treasure Island (FL): StatPearls Publishing 2021 Jan.

13. Mestdagh H, Bailleul JP, Chambon JP, Laraki A. The dorsal arterial network of the wrist with reference to the blood supply of the carpal bones. Acta Morphol Neerl Scand. 1979;17(1):73-80.

14. Rennie C, Britton J, Prouse P. Bilateral Avascular Necrosis of the Lunate in a Patient with Severe Raynaud's Phenomenon and Scleroderma. J Clin Rheumatol. 1999;5(3):165-8.

\section{Conflict of interest: none declared}

Financial support: none declared
15. Beredjiklian PK. Kienböck's Disease. J Hand Surg. 2009;34A:167175.

16. Sandler RD, Highland AM, Hughes M. Osteonecrosis of the lunate in systemic sclerosis-an underappreciated vascular complication? Clin Rheumatol. 2020;39(10):3137-3138.

17. Irisarri C. Aetiology of Kienböck's Disease. Handchir Mikrochir Plast Chir. 2010;42:157-161.

18. Zegarra-Mondragón S, Llop-Vilaltella M, Sifuentes-Giraldo WA, de la Puente Bujidos C. Osteonecrosis of the Lunate Associated with Systemic Sclerosis:Report of 4 Cases. Reumatol Clin (Engl Ed). 2019;15(5):e70-e71.

19. Goldfarb CA, Hsu J, Gelberman RH, Boyer MI. The Lichtman classification for Kienböck's disease:an assessment of reliability. $J$ Hand Surg Am. 2003;28(1):74-80.

20. Kim TG, Heo YM, Min YK. Extensor Tendon Rupture Due to Advanced Kienböck's Disease:Two Case Reports and a Review of Literature. J Hand Surg Asian Pac Vol. 2020;25(1):123-128.

21. Lichtman DM, Lesley NE, Simmons SP. The classification and treatment of Kienbock's disease: the state of the art and a look at the future. J Hand Surg Eur Vol. 2010 Sep;35(7):549-54.

22. Schuind F, Eslami S, Ledoux P.J Bone Joint Surg Br. 2008;90(2):1339.

23. Kallen AM, Strackee SD. On the history and definition of Preiser's disease. J Hand Surg Eur Vol. 2014;39(7):770-6.

24. Schmitt R, Frohner S, van Schoonhoven J, et al. Idiopathic osteonecrosis of the scaphoid (Preiser's disease)-MRI gives new insights into etiology and pathology. Eur J Radiol. 2011; 77(2):228-34.

25. Kretschmer F. Carpal tunnel syndrome induced by aseptic necrosis of the scaphoid bone. Zentralb/ Chir. 1985;110(10):6235.

26. Garg B, Gupta H, Kotwal PP. Nontraumatic osteonecrosis of the distal pole of the scaphoid. Indian J Orthop. 2011;45(2):185-187.

27. Claessen FMAP, Schol I, Kolovich GP, Ring D. Avascular Necrosis of the Scaphoid. Preiser Disease. Arch Bone Jt Surg. 2020;8(1):9498.

28. Urban MA, Green DP, Aufdemorte TB. The patchy configuration of scaphoid avascular necrosis. J Hand Surg Am. 1993;18(4):66974.

29. Rizzo M, Moran SL. Vascularized bone grafts and their applications in the treatment of carpal pathology. Semin Plast Surg. 2008;22(3):213-27. 